\title{
NUEVOS REGISTROS DE PLUTEUS FR. (BASIDIOMYCETES, AGARICALES, PLUTEACEAE) EN MÉXICO
}

\author{
Olivia Rodríguez y Laura GuZmán-Dávalos \\ Universidad de Guadalajara, Departamento de Botánica y Zoología, \\ Apdo postal 1-139, 45101 Zapopan, Jalisco, México. \\ oliviaro@cucba.udg.mx, lguzman@cucba.udg.mx
}

\section{RESUMEN}

Se suman tres nuevos registros del género Pluteus Fr. para la micobiota mexicana: Pluteus amphicystis, $P$. martinicensis y P. oligocystis. Las dos primeras especies pertenecen a la sección Pluteus y la última a la sección Celluloderma. Se describen además $P$. atromarginatus y P. salicinus, ambas de la sección Pluteus, que previamente sólo se habían listado para los estados de Jalisco e Hidalgo, respectivamente. Se registra a P. atromarginatus por primera vez para Veracruz.

Palabras clave: Agaricales, México, Pluteaceae, Pluteus.

\section{ABSTRACT}

Three species of genus Pluteus Fr., new to the Mexican mycobiota are reported: Pluteus amphicystis, $P$. martinicensis and P. oligocystis. The first two species belong to section Pluteus and the last one to section Celluloderma. Besides, $P$. atromarginatus and $P$. salicinus, both belonging to section Pluteus, are described; previously they were only known from the states of Jalisco and Hidalgo, respectively. Pluteus atromarginatus is reported for the first time from Veracruz.

Key words: Agaricales, Mexico, Pluteaceae, Pluteus.

\section{INTRODUCCIÓN}

El género Pluteus es un Agarical de la familia Pluteaceae, caracterizado por sus láminas libres, rosadas, por sus basidiosporas de pared lisa, su trama himenófora 
inversa y por ser lignícola (Banerjee y Sundberg, 1993). Este trabajo es una contribución más hacia el conocimiento del género Pluteus en México, estudio iniciado a partir de 1997 (Rodríguez y Guzmán-Dávalos, 1997, 1998, 2000, 2001; Rodríguez et al., 1997, 2004). Se definen tres nuevos registros para el país y se aportan las descripciones de dos especies adicionales que sólo se habían listado (Rodríguez et al., 2004).

\section{MATERIALES Y MÉTODOS}

Los materiales estudiados proceden tanto de bosques tropicales (tropical caducifolio, tropical perennifolio), subtropicales (mesófilo de montaña), así como de bosques templados (pino-encino y pino-abeto). Se revisaron ejemplares de herbarios mexicanos (FCME, IBUG, XAL), además de otros extranjeros (AH, GDAC, LD, WTU, ZT) para corroborar la determinación de los taxones. Para el estudio macro y micromorfológico de los especímenes considerados se siguió la metodología de Cifuentes et al. (1986), Largent (1986) y Largent et al. (1977). Para la determinación de las especies se utilizaron principalmente las obras de Pegler (1983) y Singer (1958). Las descripciones y mediciones de las estructuras microscópicas se realizaron en preparaciones montadas con hidróxido de potasio $(\mathrm{KOH})$ a 3\%. Las ilustraciones de los caracteres microscópicos examinados fueron realizadas con la ayuda de un tubo de dibujo o cámara clara. La escala de las figuras que se presentan equivale a $20 \mu \mathrm{m}$ en basidiosporas y $10 \mu \mathrm{m}$ en el resto de las estructuras observadas. Los términos empleados para las descripciones de estructuras y formas, especialmente las micromorfológicas, fueron tomados de Largent et al. (1977), Vellinga (1988), Ulloa (1991) y Kirk et al. (2001). Las abreviaturas de los herbarios están dadas de acuerdo con Holmgren et al. (1990).

\section{RESULTADOS Y DISCUSIÓN}

Descripción de las especies

Pluteus amphicystis Singer, Lloydia 21: 213, lám. 3, 1958. (Figs. 1 y 2)

Píleo de 35-55 mm de diám., convexo, umbonado, margen estriado por transparencia, granuloso en el centro (fibrillas que se agrupan dando la apariencia de 


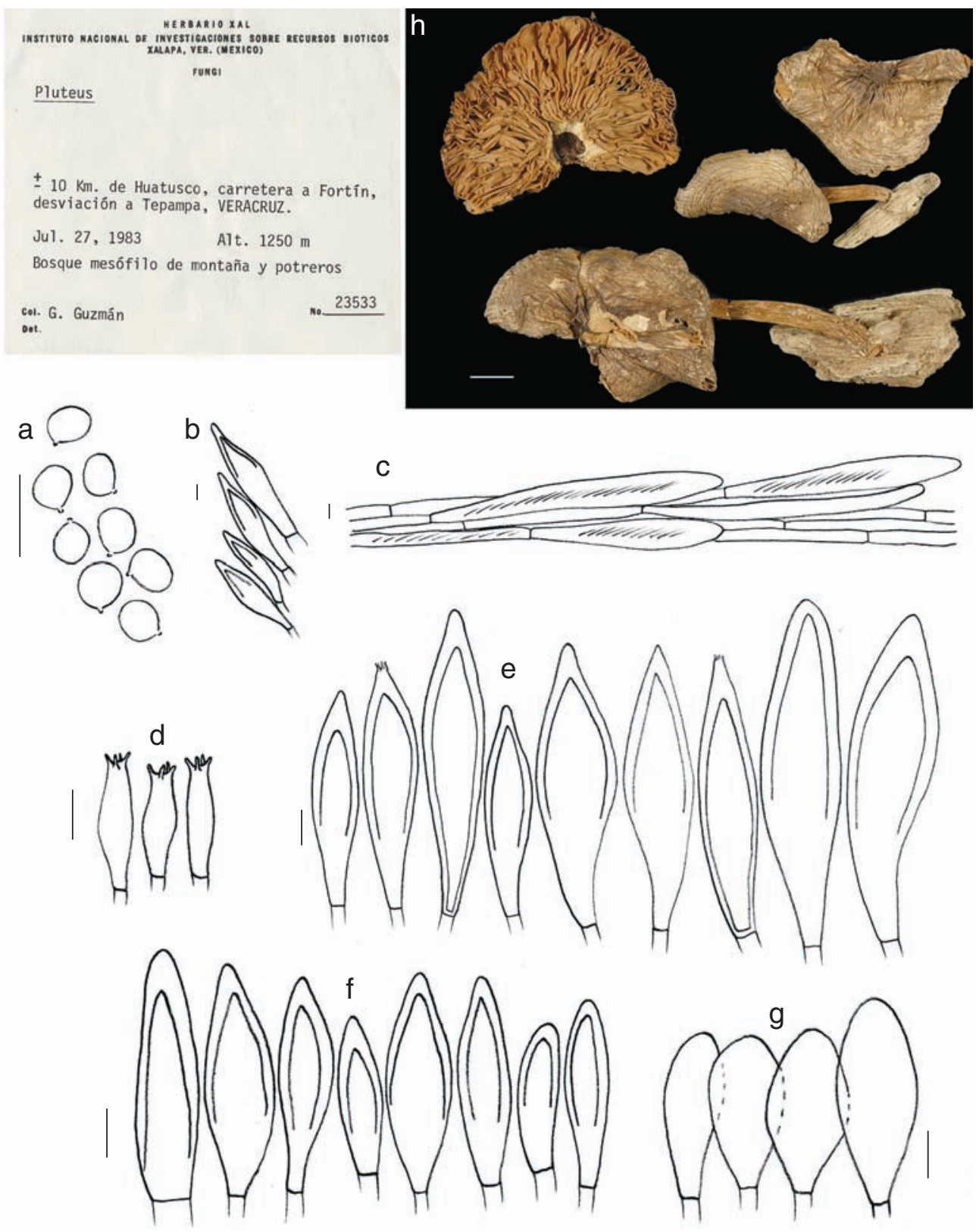

Fig. 1. Pluteus amphicystis, a. esporas; b. caulocistidios; c. pileipellis; d. basidios; e. cistidios metuloides; f. cistidios metuloides cercanos a la arista; g. queilocistidios; h. basidiomas secos (G. Guzmán 23533-XAL) (escala a = $20 \mu \mathrm{m}, \mathrm{b}-\mathrm{g}=10 \mu \mathrm{m}, \mathrm{h}=1 \mathrm{~cm}$ ). 


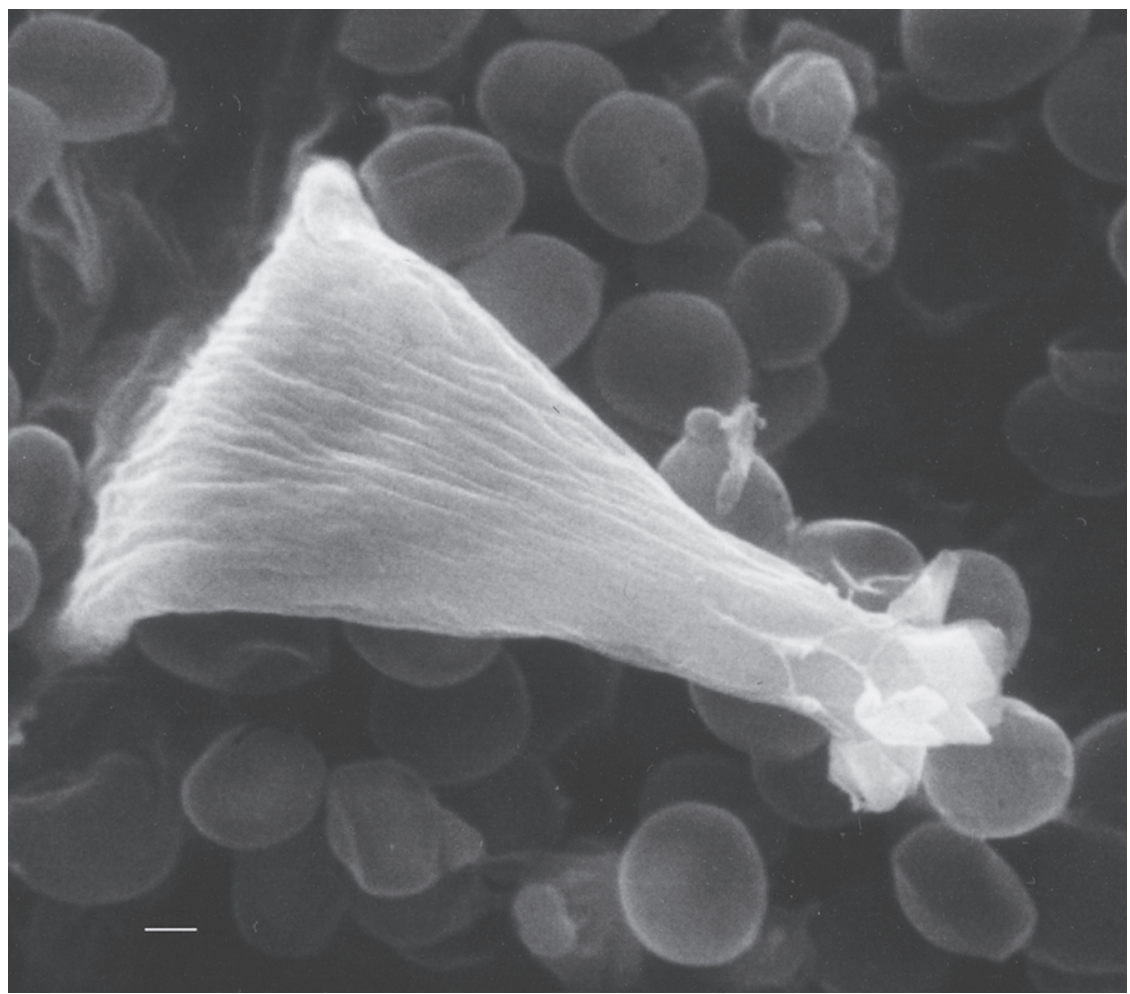

Fig. 2. Pluteus amphicystis, pleurocistidio metuloide (G. Guzmán 23533-XAL) (escala = 5 $\mu \mathrm{m})$.

gránulos, en seco) el resto liso, superficie seca, color café a café-amarillo-yodo a finalmente café-rosado pálido en el margen. Láminas libres, apretadas, ventricosas, de color café-rosado, arista entera, concolora. Estípite de 35-80 x 2-6 mm, central, cilíndrico, base algo ensanchada, ligeramente torcido, liso a granuloso-flocoso hacia la base, amarillo (Fig. 1h). Contexto blanco a amarillo. Olor desagradable.

Basidiosporas de 4.5-6.5 x 4-5 (-5.5) $\mu \mathrm{m}$, en promedio de 5 x $4 \mu \mathrm{m}, \mathrm{Q}=1.1-$ 1.25, subglobosas a anchamente elipsoides, lisas, de pared delgada a subgruesa, con contenido refringente, hialinas. Basidios de 25-29 x 6-8 $\mu \mathrm{m}$, tetraspóricos, claviformes, hialinos (Fig. 1d). Pleurocistidios metuloides tipo magnus, de 44-88 (-101) $\mathrm{x}$ (10-) 14-24 (-27) $\mu \mathrm{m}$, ventricosos o sublageniformes, con pared de hasta $4 \mu \mathrm{m}$ de grosor, con finas incrustraciones de cristales en el ápice, hialinos (Figs. 1e y 2); cerca de la arista también presentes pero sin incrustración y de tamaño más pequeño, de 32-43 x 9-15 $\mu \mathrm{m}$, ventricoso-fusiformes, pared de hasta $3 \mu \mathrm{m}$ de grosor principal- 
mente hacia el ápice (Fig. 1f). Queilocistidios de 35-43 x 12-18 $\mu$ m, claviformes con el ápice obtuso a ligeramente atenuado, de pared delgada, hialinos (Fig. 1g). Pileipe1lis en cutis, con elementos terminales de 65-80 x 11.2-20 (-25) $\mu \mathrm{m}$, filamentosos, de ápice anchamente redondeado, de pared delgada pero en algunos con engrosamiento en el ápice, hialinos o con contenido de color café-amarillento, sin fíbulas, postrados, algunos suberectos cerca del centro del píleo (Fig. 1c). Caulocistidios de 42.5-70 x 7.5-15 $\mu \mathrm{m}$, de tipo metuloide, fusiforme-ventricosos, de pared gruesa (hasta $2 \mu \mathrm{m}$ ), hialinos, observados hacia la base del estípite, sin fíbulas (Fig. 1b).

Hábitat. Lignícola, gregario, en potrero en medio de bosque mesófilo de montaña.

Espécimen examinado. VERACRUZ: Mpio. de Huatusco, $\pm 10 \mathrm{~km}$ de Huatusco, carretera a Fortín, desviación a Tepampa, 1250 m s.n.m., 27.VII.1983, G. Guzmán 23533 (XAL).

Observaciones. De acuerdo con Singer (1958), Pluteus amphicystis se distingue por la coloración amarilla del basidioma y los cistidios metuloides, que carecen de cuernos apicales o prolongaciones laterales, características por las que incluyó a esta especie en la estirpe Amphicystis de la sección Pluteus. Según Pegler (1983), el píleo es de color ante-ocráceo claro, que coincide con lo observado en el material estudiado.

El espécimen de Veracruz concuerda en general con lo descrito en la literatura, a excepción de los queilocistidios, que Singer (op. cit.) registró más pequeños, de 23-28 x 10-20 $\mu \mathrm{m}$, y vesiculoso-subclaviformes. Por su parte, Pegler (1983) no los observó, ya que lo que describió (40-80 x 16-26 $\mu \mathrm{m}$, ventricoso-fusoides, de pared gruesa) corresponde a los pleurocistidios, que ocasionalmente se pueden observar en la arista de la lámina. Por otro lado, los pleurocistidios observados en el material mexicano son ligeramente mayores con respecto a lo descrito por varios autores:

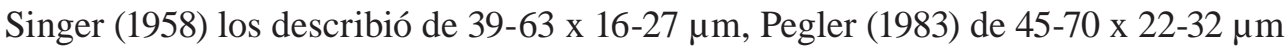
y Pradeep y colaboradores (2002) de 60-81 x 13.5-22.5 $\mu \mathrm{m}$.

La pileipellis de Pluteus amphicystis fue interpretada por Pradeep et al. (2002) como una tricodermis, lo que no concuerda con lo registrado por Singer (op. cit.) y Pegler (op. cit.). Estos últimos autores la describieron como "una epicutis" de hifas postradas radiales con elementos terminales dermatocistidioides principalmente en el centro del píleo.

Pluteus reticulatus Murrill es afín a $P$. amphicystis por el tipo de cistidios metuloides y la coloración miel pálida del estípite, pero difiere por el píleo velutino y por los queilocistidios lanceolado-angostos (Pegler, 1968). 
Otros taxones como Pluteus horridus Singer y P. spinulosus Murrill son parecidos a $P$. amphicystis en la presencia de los cistidios metuloides sin cuernos; sin embargo, se diferencian por el color de los basidiomas, los cistidios metuloides con espinas o prolongaciones laterales, así como por el tamaño de las basidiosporas, de 6-8 x 5-6.5 $\mu \mathrm{m}$, y la presencia de fíbulas en la pileipellis (Murrill, 1917; Singer, 1973). Pluteus magnus McClatchie es también afín a P. amphicystis en los cistidios metuloides sin cuernos, pero éstos son escasos, además las basidiosporas y queilocistidios son de mayor tamaño ((4-)5-7.4 x 4.6-5.8(-6.5) $\mu \mathrm{m}$ y (22-) 45-80 (-120) x (8-).12-24 $\mu \mathrm{m}$, respectivamente) (Banerjee y Sundberg, 1995). Pluteus amphicystis es una especie escasa, hasta ahora conocida de Bolivia (Singer, 1958), India (Pradeep et al., 2002) y La Martinica (Pegler, 1983) y citada aquí como nuevo registro de México.

Pluteus atromarginatus (Singer) Kühner, Bull. Mens. Soc. Linn. Lyon 4: 5, 1935. (Fig. 3)

$\equiv$ P. cervinus var. atromarginatus Singer, Z. Pilzk. 4: 40, 1925.

$\equiv$ P. cervinus subsp. atromarginatus (Singer) Konrad, Bull. Soc. Mycol. France 42: 148, 1927.

Píleo de 30-70 mm de diám., convexo, subumbonado, margen estriado, superficie finamente fibrilosa, higrófana, centro de color café-chocolate-negruzco, el resto café oscuro. Láminas libres, apretadas, ventricosas, de color rosado, arista irregularmente marginada, finamente fimbriada, café oscura. Estípite de 51-70 x 4-8 $\mathrm{mm}$, central, cilíndrico, fibriloso, brillante, blanquecino, manchado en la superficie de color pardusco al roce. Contexto de hasta $8 \mathrm{~mm}$ en la parte media del píleo, blanquecino (Fig. 3f). Olor a humedad y sabor inapreciable.

Basidiosporas de 5.5-8 (-8.5) x 4-6 $\mu \mathrm{m}$, en promedio de $6.5 \times 5 \mu \mathrm{m}, \mathrm{Q}=$ 1.13-1.62, anchamente elipsoides a elipsoides, lisas, de pared delgada, con contenido granular, hialinas (Fig. 3a). Basidios de 24.8-37 (-42) x 7.5-9.5 $\mu \mathrm{m}$, tetraspóricos, claviformes, con contenido granuloso, hialinos (Fig. 3c). Pleurocistidios metuloides tipo cervinus, de 53.6-96 x 11.2-26 $\mu \mathrm{m}$, fusoide-ventricosos, con 2-4 cuernos, algunos bifurcados, con pared hasta de $2 \mu \mathrm{m}$ de grosor hacia la parte apical, con contenido de color café-amarillento (Fig. 3d). Queilocistidios de (28.8-) 37-63.2 x 12-27 $\mu \mathrm{m}$, claviformes, con pigmento intracelular de color café, con fíbulas, agrupados en fascículos (Fig. 3e). Pileipellis en cutis, con elementos de 87.5-130 x 5.6-12.5 $\mu \mathrm{m}$, filamentosos, con ápices redondeados, de pared delgada, con contenido intracelular homogéneo de color café intenso, fíbulas presentes (Fig. 3b). Estipitipellis con hifas 


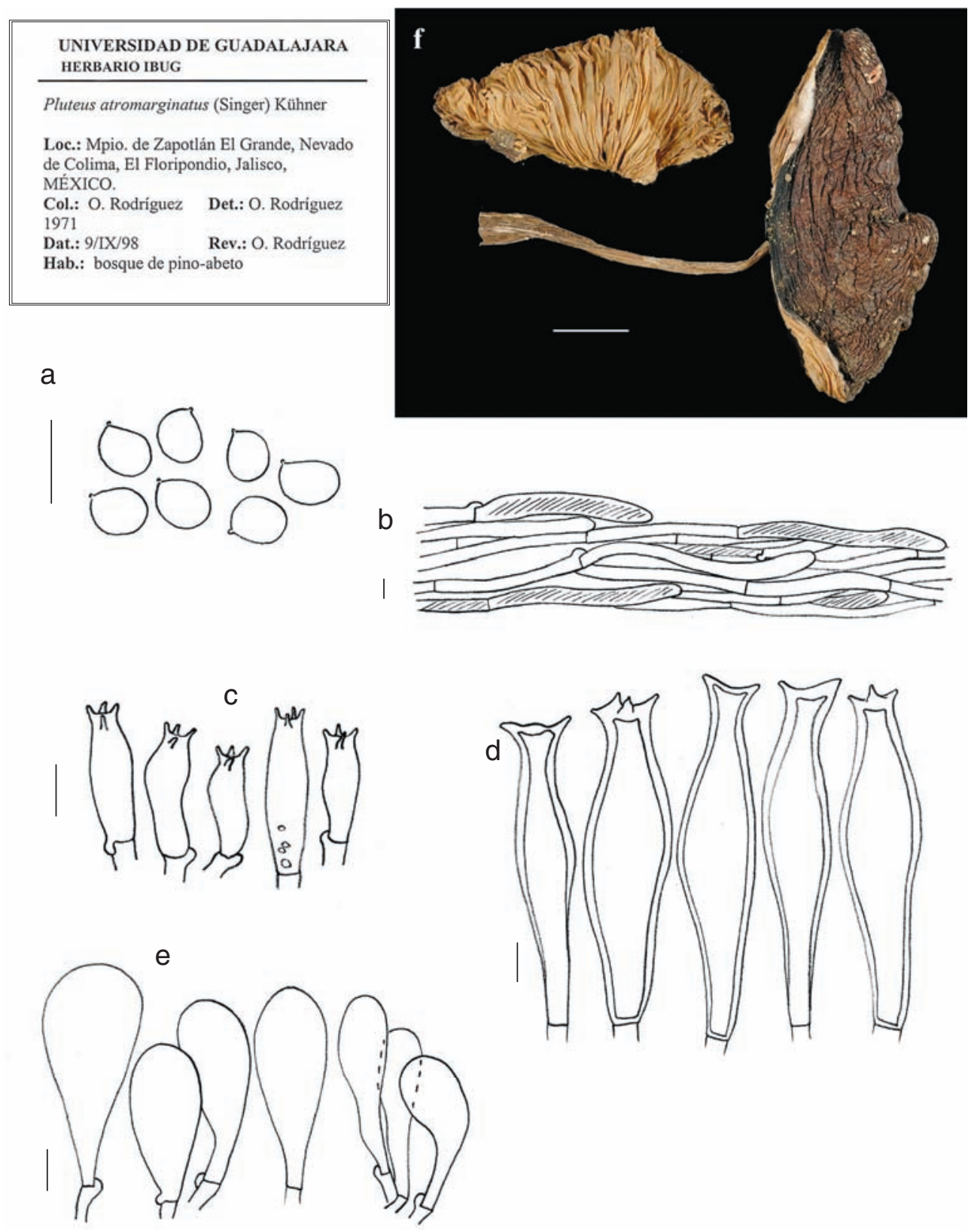

Fig. 3. Pluteus atromarginatus. a. esporas; b. pileipellis; c. basidios; d. cistidios metuloides tipo cervinus; e. queilocistidios; f. basidiomas secos (O. Rodríguez 1971-IBUG) (escala a = $20 \mu \mathrm{m}, \mathrm{b}-\mathrm{e}=10 \mu \mathrm{m}, \mathrm{f}=1 \mathrm{~cm}$ ). 
de 4-13.6 $\mu$ m de diám., hialinas o de color café-amarillento, fíbulas observadas hacia la base del estípite.

Hábitat. Lignícola, solitario, en bosques de pino-encino y de pino-abeto.

Especímenes examinados. JALISCO: Mpio. de Zapotlán el Grande, Nevado de Colima, El Floripondio, 5.IX.1992, G. Guzmán 30554 (XAL); Ibid., 2000 m s.n.m., 9.IX.1998, O. Rodríguez 1971 (IBUG). VERACRUZ: Mpio. de Xico, parte Este del Cofre de Perote, Los Gallos, Ej. Ingenio El Rosario, 19.VI.1986, L. Villarreal 2486 (XAL).

Material adicional estudiado. ESPAÑA: Prov. Guadalajara, entre Condemios de Abajo y Aldeanueva de Atienza, bajo Pinus sylvestris, 4.X.1991, M. Heykoop y G. Moreno s.n. (AH-13856); Prov. Madrid, Dehesa de Cercedilla, 13.XI.2004, G. Moreno s.n. (AH). E.U.A.: Washington, Seattle, University of Washington, D. E. Stuntz 520, 9513 (WTU). ITALIA: Consenza, 800 m s.n.m., 28.X.1985, C. Lavorato s.n. (ZT-851028-28); La Sila, Consenza, 900 m s.n.m., 2.XI.1994, C. Lavorato s.n. (ZT-941102-28). RUMANIA: Tg. Neamt, 670 m s.n.m., 7.VIII.1967, Th. Chifu s.n. (ZT-198).

Observaciones. Los materiales estudiados concuerdan con lo citado por Banerjee y Sundberg (1995), Breitenbach y Kränzlin (1995), Orton (1986) y Vellinga (1990). Pluteus atromarginatus es un hongo con basidiomas generalmente grandes y robustos, en los que el píleo puede llegar a medir $120 \mathrm{~mm}$ de diámetro o más. Se considera una especie fácil de distinguir por sus láminas marginadas, con la arista de color café, que se observa aun en estado seco. En cuanto a caracteres micromorfológicos, se caracteriza por la presencia de pleurocistidios metuloides, que la incluyen en la sección Pluteus y que la diferencian fácilmente de otras especies macroscópicamente afines, al igual que por mostrar fíbulas en la pileipellis, aunque no en todos los septos.

El pigmento de color café típico de los queilocistidios, fue observado además en los pleurocistidios. Esta coloración sólo había sido citada en los primeros, y es la que origina la arista marginada en este caso. El tamaño de las basidiosporas fue ligeramente mayor con respecto a las colecciones europeas estudiadas y a lo mencionado en la bibliografía revisada, que en promedio las registraron de (4.8-) 5.5-7.5 (-8) x (3.6-) 4-5.5 $\mu \mathrm{m}$; no obstante en Norteamérica, Banerjee y Sundberg (1995) las consignaron como de 6-7.8 (-9) x 4.4-5.6 $\mu \mathrm{m}$.

La existencia de fíbulas en las hifas del estípite es un carácter poco citado en esta especie, sólo registrado por Vellinga (1990), quien las observó muy abundantes en todos los tejidos del basidioma. En los ejemplares mexicanos y otros materiales aquí estudiados se les observó, aunque como poco frecuentes, generalmente hacia la base del estípite. 
Al estudiar los especímenes examinados se encontró que en general existe homogeneidad en el tamaño de las estructuras microscópicas para esta especie, como en el caso de los pleuro y queilocistidios. La variación fue mínima entre lo observado en los ejemplares mexicanos con respecto a los europeos, en comparación con otros taxones, en donde se han encontrado mayores diferencias. Consideramos que Pluteus atromarginatus es una especie muy bien definida y poco variable.

Taxones relacionados con Pluteus atromarginatus son P. cervinus (Schaeff.) P. Kumm. y P. pouzarianus Singer, los cuales no presentan las láminas marginadas y tampoco fíbulas (Breitenbach y Kränzlin, 1995). Otra especie afín es $P$. umbrosus (Pers. : Fr.) P. Kumm., que se parece a $P$. atromarginatus por tener la arista de las láminas de color café; no obstante, no está incluida en la sección Pluteus ya que carece de cistidios metuloides y presenta el píleo y estípite fuertemente escamosos (Orton, 1986; Vellinga, 1990).

Pluteus atromarginatus se encuentra regularmente sobre madera de coníferas; en cambio, se cita pocas veces de árboles caducifolios. Es una especie no muy común pero de una amplia distribución, registrada hasta ahora de Norteamérica, Asia, Europa (Breitenbach y Kränzlin, 1995) y recientemente citada para la micobiota mexicana (Rodríguez et al., 2004).

Pluteus martinicensis Singer \& Fiard in Pegler, Kew. Bull. Add. Ser. 9: 311, 1983. (Fig. 4)

Píleo de 15-27 mm diám., convexo, umbonado, margen incurvado, algo ondulado, liso a radialmente fibriloso-innato, de color café oscuro en el centro a café claro hacia el margen. Láminas libres, subapretadas, anchas, de color blanco-rosado, arista entera, concolora. Estípite de 30-40 x 1-2 mm, central, cilíndrico, algo torcido, superficie velutina hacia la base, de color blanco-grisáceo a café oscuro (Fig. 4g). Contexto blanquecino.

Basidiosporas de 6-7.6 (-8) x (5.2-) 5.6-6.8 (-7.6) $\mu \mathrm{m}$, en promedio de 6.5 x 6 $\mu \mathrm{m}, \mathrm{Q}=1.05-1.18$, subglobosas a anchamente elipsoides, lisas, de pared delgada, con contenido granular, hialinas (Fig. 4a). Basidios de 25-26 x 7-8 $\mu \mathrm{m}$, tetraspóricos, claviformes, hialinos (Fig. 4e). Pleurocistidios metuloides de tipo cervinus, de (59-) 77.6-96 (-137.6) x 19.2-28 $\mu \mathrm{m}$, fusoide-ventricosos, con 2-3 (-4) cuernos, agudos, algunos bifurcados, con pared de $1 \mu \mathrm{m}$ en la parte media hasta $2 \mu \mathrm{m}$ de grosor hacia la parte apical, hialinos (Fig. 4d). Queilocistidios de 37.6-48 (-61.6) x 11.614.8 (-20.8) $\mu \mathrm{m}$, claviformes, algunos con el ápice ligeramente atenuado, hialinos (Fig. 4f). Pileipellis en cutis, con elementos de 75-112.5 (-140) x 8.8-14.4 $\mu \mathrm{m}$, en su 

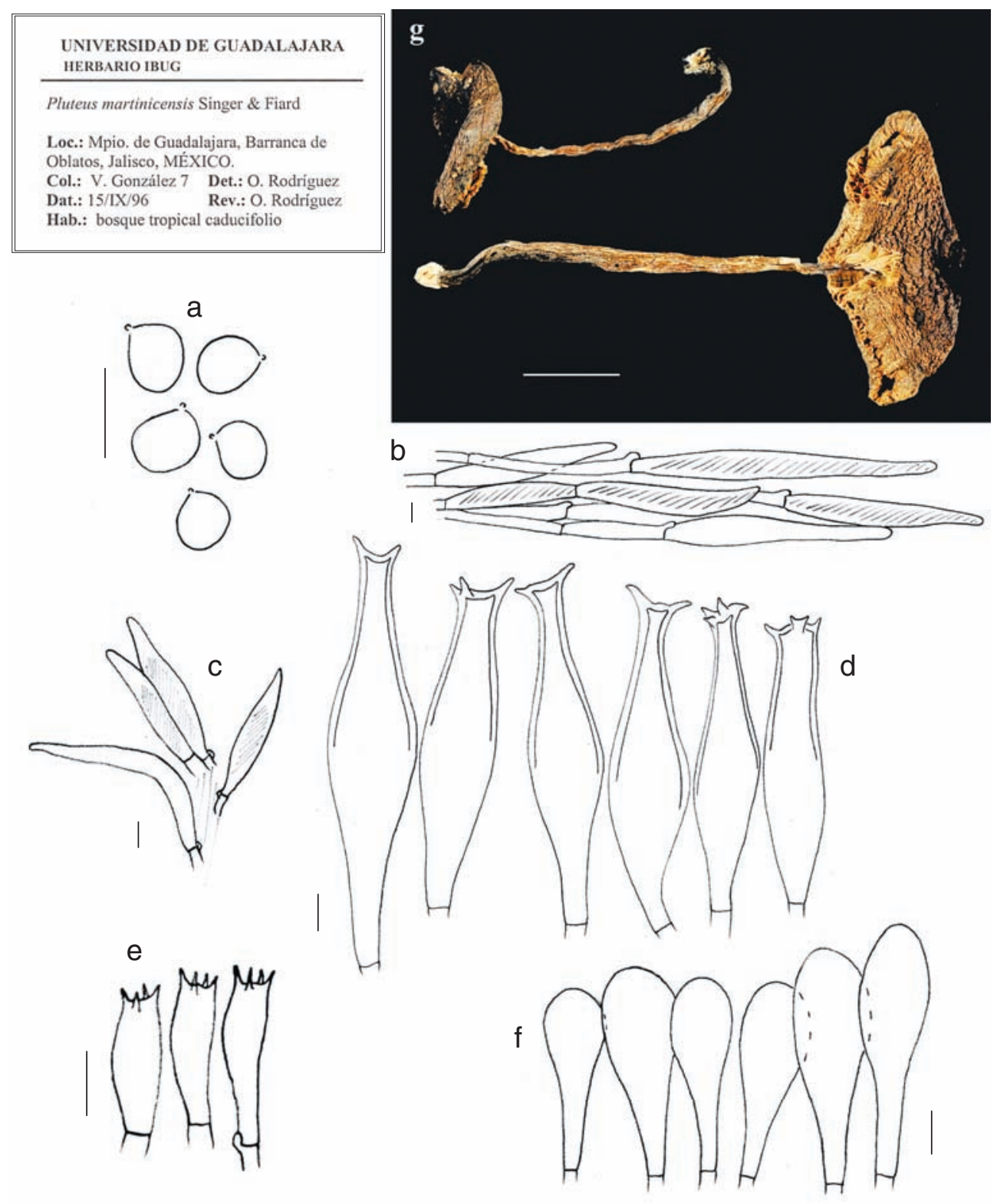

Fig. 4. Pluteus martinicensis. a. esporas; b. pileipellis; c. caulocistidios; d. cistidios metuloides tipo cervinus; e. basidios; f. queilocistidios; g. basidiomas secos (V. González 7-IBUG) (escala $\mathrm{a}=20 \mu \mathrm{m}, \mathrm{b}-\mathrm{f}=10 \mu \mathrm{m}, \mathrm{g}=1 \mathrm{~cm})$. 
mayoría subfusiformes a subcilíndricos, con contenido intracelular homogéneo de color café intenso, fíbulas presentes (Fig. 4b). Caulocistidios de 55-85 x 7.5-11.2 $\mu \mathrm{m}$, subfusiformes, más o menos similares a lo observado en la pileipellis, con pigmento de color café-amarillento, fíbulas presentes, agrupados en fascículos (Fig. 4c).

Hábitat. Lignícola, gregario, en bosque tropical caducifolio muy perturbado.

Espécimen examinado. JALISCO: Mpio. de Guadalajara, $260 \mathrm{~m}$ de la entrada a la Barranca de Oblatos, 15.IX.1996, V. González 7 (IBUG).

Observaciones. El material mexicano concuerda con la descripción original de Pluteus martinicensis, excepto en que Pegler (1983) mencionó un tamaño menor (5-7 x 4.5-6 $\mu \mathrm{m}$ ) de las basidiosporas; sin embargo, las medidas de basidiosporas observadas por Pradeep et al. (2002) coinciden con las del material aquí estudiado.

Singer y Fiard (en Pegler, 1983) describieron caulocistidios fasciculados sobre toda la superficie del estípite, característica que distingue a la especie. Pradeep et al. (2002) consideraron otras particularidades de P. martinicensis, como son el píleo de color café, la superficie fibrilosa-estriada, la arista laminar coloreada y la presencia de fíbulas en la pileipellis y estipitipellis. Este último carácter no fue observado en el revestimiento del estípite por Singer y Fiard (Pegler, 1983), quienes además mencionaron que la coloración de la arista de las láminas puede o no estar presente, lo que coincide con el espécimen mexicano, en el que tal rasgo no se observó y en el que los queilocistidios son hialinos. La descripción de Pradeep et al. (op. cit.) tiene otras pequeñas diferencias con el material mexicano, en el tamaño del píleo (50-95 $\mathrm{mm}$ ) y de los pleurocistidios (45-81 x 15-22.5 $\mu \mathrm{m}$ ), principalmente.

Pluteus triplocystis Singer, descrito de México (Singer, 1973), es afín a $P$. martinicensis por presentar caulocistidios fasciculados, fusiformes o subfusiformes; sin embargo, se separa por tener sus basidiosporas más pequeñas (5.5-7.5 x 4.5-5.5 $\mu \mathrm{m}$ ) y globosas, cistidios metuloides más inflados y con cuernos cortos, así como por la presencia de fíbulas en todas sus estructuras micromorfológicas (Singer, 1973). Pluteus martinicencis se considera una especie escasa, hasta ahora citada de La Martinica y la India (Pegler, 1983; Pradeep et al., 2002). Es un nuevo registro para la micobiota mexicana.

Pluteus oligocystis Singer, Lloydia 21(4): 266, 1958. (Fig. 5)

Píleo de 6 mm de diám., convexo-aplanado, umbonado, margen rimoso, superficie pruinosa-subpubescente, de color café muy oscuro. Láminas libres, apretadas, anchas, de color rosado, arista fimbriada, más clara. Estípite de 9 x $0.5 \mathrm{~mm}$, 

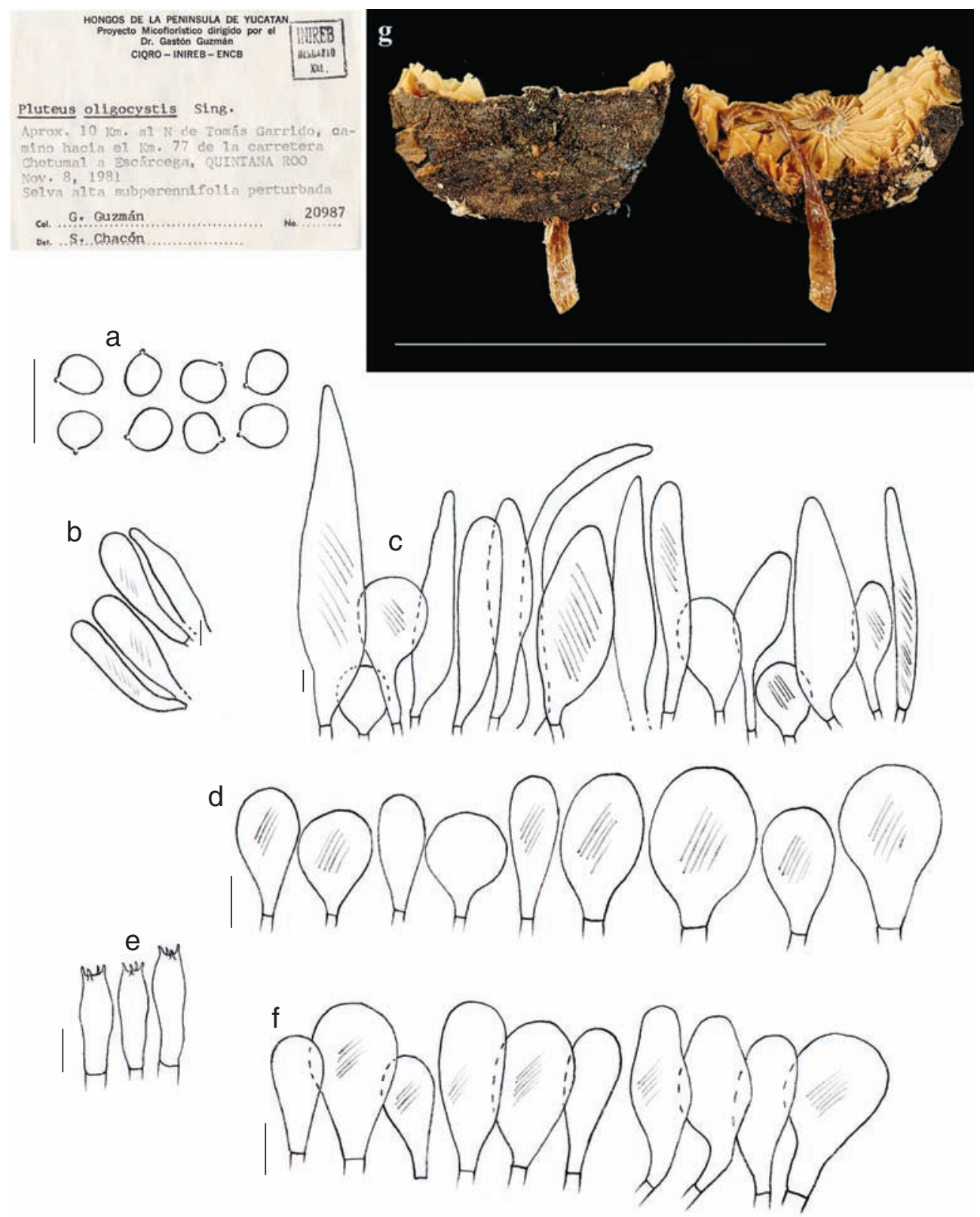

Fig. 5: Pluteus oligocystis. a. esporas; b. caulocistidios; c. pileipellis; d. pleurocistidios; e. basidios; f. queilocistidios; g. basidiomas secos (G. Guzmán 20987-XAL) (escala a = 20 m, b-f $=10 \mu \mathrm{m}, \mathrm{g}=1 \mathrm{~cm})$. 
central, más o menos cilíndrico, glabro, sedoso, de color café pálido, con micelio blanquecino en la base (Fig. 4g).

Basidiosporas de 4.5-5.6 x 4.3-5 $\mu \mathrm{m}$, en promedio de 5 x $4 \mu \mathrm{m}, \mathrm{Q}=1.08-1.3$, anchamente elipsoides a subglobosas, lisas, de pared delgada, con contenido refringente, hialinas (Fig. 5a). Basidios de 25-27 x 7-8 $\mu \mathrm{m}$, tetraspóricos, claviformes, hialinos, fácilmente colapsables, escasos (Fig. 5e). Pleurocistidios de 20-32.5 x 8.7-20.6 $\mu \mathrm{m}$, claviformes, elipsoides o subglobosos, de pared delgada, hialinos o con contenido intracelular de color café-amarillento, no muy abundantes (Fig. 5d). Queilocistidios de 23.7-37.5 x 10.6-20 $\mu \mathrm{m}$, claviformes, subglobosos u obovoides, algunos subutriformes, de pared delgada, hialinos o con contenido intracelular de color caféamarillento (Fig. 5f). Pileipellis de tipo mixtini, pileocistidios de 86.4-153.8 x (7.6-) 15.3-30.7 $\mu \mathrm{m}$, largamente fusiformes o alargado-cilíndricos a fusiforme-ventricosos, sublageniformes, con ápices obtusos, de pared delgada, de color café oscuro mezclados con elementos de 27.6-61.5 x 15.3-30.7 $\mu \mathrm{m}$, globosos o subglobosos, esferopedunculados, claviformes o angostamente claviformes, de pared delgada, con pigmento condensado de color café oscuro (Fig. 5c). Caulocistidios de 41.5-56.9 x 7.6-13.8 $\mu \mathrm{m}$, angostamente claviformes a claviforme-cilíndricos, de pared delgada, con contenido intracelular pigmentado de color café-amarillento (Fig. 5b).

Hábitat. Lignícola, solitario, en bosque tropical perennifolio perturbado.

Espécimen examinado. QUINTANA ROO: Mpio. de Othón Pompeyo Blanco, aproximadamente $10 \mathrm{~km}$ al N de Tomás Garrido, camino hacia el km 77 de la carretera Chetumal a Escárcega, 8.XI.1981, G. Guzmán 20987 (IBUG).

Observaciones. Pluteus oligocystis se caracteriza por el tipo de pileipellis y los pleurocistidios escasos o ausentes. El material mexicano concuerda en general con la descripción de Singer (1958), excepto el tipo de queilocistidios que los describió como “oblongo-ventricosos mucronados o con constricción”, formas que no se han observado en la recolección estudiada.

Entre los taxones estrechamente relacionados con la especie en discusión se encuentra Pluteus dennisii Singer, que Singer (1989) describió con el estípite con pequeñas granulaciones oscuras, la arista laminar blanca y los queilocistidios hialinos. El mismo autor (1958) mencionó que existe afinidad entre $P$. oligocystis y $P$. variipes var. variipes Singer, esta última diferenciada por la pileipellis que presenta tres tipos de elementos y las basidiosporas ligeramente más pequeñas (4.5-6.2 x 4-4.8 $\mu \mathrm{m})$.

La determinación del ejemplar mexicano como $P$. oligocystis se realiza con base en la diagnosis y la descripción de la especie y se confirma de manera comparativa con las observaciones realizadas por el Dr. Horak (com. personal). 
Se cita como nuevo registro para la micobiota del país. Se considera importante obtener nuevas recolecciones para ampliar la descripción de la especie y el conocimiento de su distribución en México.

Pluteus salicinus (Pers. : Fr.) P. Kumm., Führ. Pilzk.: 99, 1871. (Fig. 6)

三Agaricus salicinus Pers., Icon. descr. Fung.: 9, 1798.

$\equiv$ Agaricus salicinus Pers.: Fr., Syst. Mycol. 1: 202, 1821.

Píleo de 20 mm de diám., plano, irregular, ligeramente deprimido, margen estriado, superficie escamoso-escabrosa en el centro a finamente fibrilosa en el resto, de color café oscuro (en seco) en el centro a grisáceo con tonos verde-grisáceos el resto. Láminas libres, apretadas, anchas, de color rosado, arista entera, concolora. Estípite de 18 × $2 \mathrm{~mm}$, central, cilíndrico, sedoso, brillante, finamente fibriloso, frágil, blanco pero manchado de tonos azul-verdosos, con micelio blanquecino en la base (Fig. 6f). Contexto no observado.

Basidiosporas de 6-8.4 x 5.2-6.8 $\mu \mathrm{m}$, en promedio de 7 × $6 \mu \mathrm{m}, \mathrm{Q}=1.05$ 1.3, subglobosas a anchamente elipsoides, lisas, de pared delgada, hialinas (Fig. 6a). Basidios de 25.6-34.4 x 6.8-9.2 $\mu \mathrm{m}$, tetraspóricos, claviformes, algunos con base ancha, con contenido granuloso, hialinos (Fig. 6c). Pleurocistidios metuloides de tipo cervinus, de (44-) 51.2-79.2 x (10.8-) 12.4-20 $\mu$ m, fusoide-ventricosos, con 2 o 3 cuernos, comúnmente cortos, más o menos obtusos o bifurcados, rectos, con pared de hasta $1.5 \mu \mathrm{m}$ de grosor hacia el ápice a partir del cuello, hialinos (Fig. 6e). Queilocistidios de (25.6-) 30.4-45.6 (-55.2) x (10-) 12.8-17.2 $\mu \mathrm{m}$, claviformes a anchamente claviformes, de pared delgada, hialinos, con fíbulas en la base (Fig. 6d). Pileipellis en cutis, con elementos de 49.2-118.2 (-157.6) x 6-14.7 $\mu \mathrm{m}$, cilíndricos, de ápices redondeados a subagudos, hialinos o con contenido de color café, con fíbulas abundantes (Fig. 6b).

Hábitat. Lignícola, solitario, en bosque mesófilo de montaña.

Espécimen examinado. HIDALGO: Mpio. de Tenango de Doria, 24 km de la desviación a Tenango de Doria, 6.VII.1980, J. Cifuentes 426 (FCME).

Material adicional estudiado. DINAMARCA: Fakse Ladeplats, 23.IX.1977, E. Horak s.n. (ZT-77/258). ESPAÑA: Prov. Huelva, Alajar, 21.XI.1991, A. Ortega s.n. (GDAC-36655); Prov. Cádiz, carretera del Puerto de Galis a Alcalá de los Gazules, 12.XII.1990, A. Ortega s.n. (GDAC-36216); sin localidad, E. Horak (ZT-6161). SUECIA: Kiaby, Ivö, Nordostsidan avön, Bokskog, 10.X.1942, O. Andersson s.n. (LD). SUIZA: Zumikon (Cantón de Zurich), Rietacher, 10.X.2003, O. Rodríguez 2562 (IBUG). 

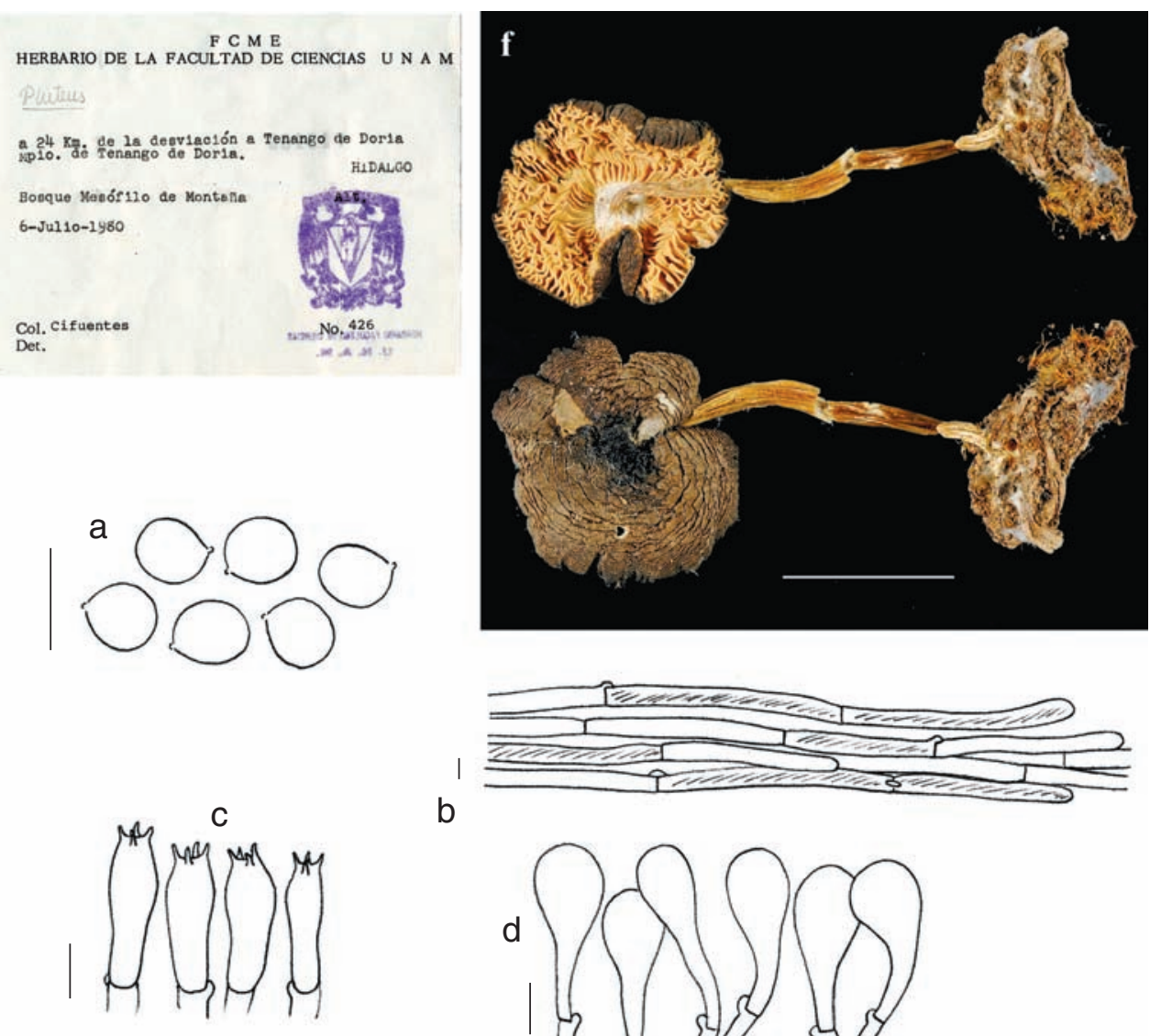

b
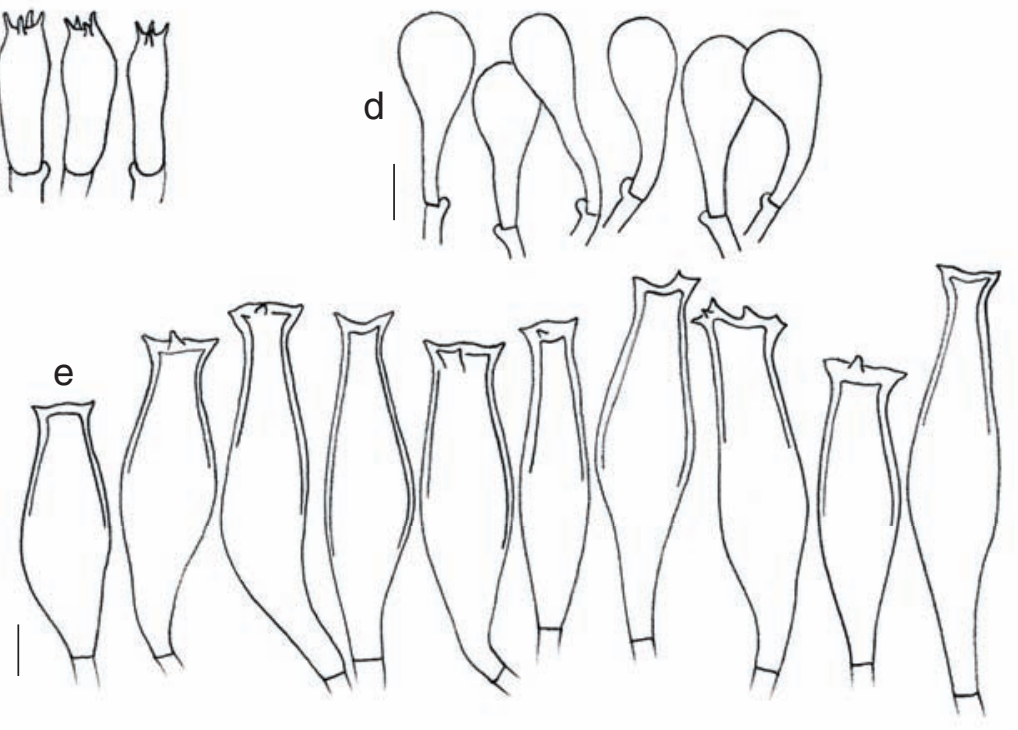

Fig. 6: Pluteus salicinus. a. esporas; b. pileipellis; c. basidios; d. queilocistidios; e. cistidios metuloides tipo cervinus; f. basidiomas secos (J. Cifuentes 426-FCME) (escala a $=20 \mu \mathrm{m}$, b-e $=10 \mu \mathrm{m}, \mathrm{f}=1 \mathrm{~cm})$. 
Observaciones. Este taxon está incluido en la sección Pluteus por presentar cistidios metuloides y una pileipellis de tipo cutis con presencia de fíbulas. En particular la especie se caracteriza por el píleo generalmente pequeño y de color gris-glauco (Lange, 1936). La presencia de fíbulas en la base de algunas estructuras, como los queilocistidios, no había sido registrada anteriormente, carácter hasta ahora sólo observado, o por lo menos mencionado, en las hifas de la pileipellis.

El tamaño de los cistidios metuloides del ejemplar estudiado concuerda con el citado por Banerjee y Sundberg (1995) para Pluteus salicinus en Norteamérica ((40-) 50-70 (-88) x (11-) 15-18 $\mu \mathrm{m})$, aunque Breitenbach y Kränzlin (1995) los registraron ligeramente más grandes con base en materiales europeos (80-95 x 16-27 $\mu \mathrm{m})$. No obstante, en las recolecciones europeas revisadas se encontraron pleurocistidios de 55-88 (-103) x (10.4-) 12-21 (-25.6) $\mu \mathrm{m}$, que se acercan a las medidas observadas por Consiglio (1999), quien las registró de 65-95 x 15-25 $\mu \mathrm{m}$.

La ausencia de los cistidios metuloides tipo magnus podría ser un carácter taxonómico a considerar dentro de la sección Pluteus, ya que en especies como la que aquí se discute, tales estructuras no fueron observadas en el material mexicano ni en todas las recolecciones europeas examinadas. En general los cistidios metuloides de tipo cervinus encontrados en este taxon son de pared no muy gruesa, aun hacia el ápice, y sus cuernos son comúnmente cortos y algo obtusos o bifurcados. Asimismo, la base de estos cistidios es corta o lleva un pedicelo muy largo, carácter muy variable, no sólo en $P$. salicinus, sino también en el resto de los taxones de esta sección, por lo que no se puede considerar para la diferenciación de especies.

Existen tres variedades de Pluteus salicinus, la típica, la variedad achloes Singer y la variedad americanus P. Banerjee \& Sundb. La var. achloes no presenta tonalidades azul-verdosas en el basidioma, y la var. americanus se distingue por el píleo liso, con margen estriado-traslúcido y por las basidiosporas de mayor tamaño [8-10 (-11) x 6.4-7.4 $\mu \mathrm{m}$ ] (Banerjee y Sundberg, 1993). El material mexicano concuerda más con Pluteus salicinus var. salicinus, por la coloración azul-verdosa en el estípite y el píleo, la superficie del píleo escamosa en el centro y por el tamaño de las basidiosporas. Es interesante mencionar que Pluteus salicinus desarrolla una coloración azul-verdosa al rozar el basidioma, debido a la presencia de psilocibina y psilocina (Saupe, 1981; Stijve y Bonnard, 1986; Stijve, 1995; Gartz, 1996).

Pluteus cinereofuscus J. E. Lange es afín a P. salicinus, ya que presenta tonalidades café-grisáceas sobre el píleo, pero se distingue por su pileipellis de tipo himenodermis, pleurocistidios de pared delgada y por fructificar en madera de coníferas (Breitenbach y Kränzlin, 1995). Cuando tiene tonos grises en el píleo, $P$. salicinus se puede confundir con $P$. cervinus, del que se diferencia por la ausencia 
de fíbulas en la pileipellis y en el hábitat, ya que generalmente crece en madera de coníferas (Breitenbach y Kränzlin, op. cit.).

Pluteus salicinus se considera una especie poco frecuente, conocida de Europa, Norteamérica, Asia y norte de África (Breitenbach y Kränzlin, 1995) y recientemente registrada del centro de México (Rodríguez et al., 2004).

\section{AGRADECIMIENTOS}

Se reconoce a las autoridades de la Universidad de Guadalajara por el apoyo otorgado a la primera autora para la realización de este trabajo y de sus estudios de doctorado. Se recibió ayuda económica del Consejo Nacional de Ciencia y Tecnología (proyecto CONACYT-SEP-2003-C02-42957); del Programa de Mejoramiento del Profesorado de Educación Superior a través de su programa de apoyo a ex-becarios a la segunda autora, proyecto PROMEP/103.5/03/2580, y de la Universidad de Guadalajara, proyectos P3E 34961 y 50052 del Cuerpo Académico CA-23. Se agradece al Dr. E. Horak del Geobotanisches Institut, Eidgenossische Technische Hochschule, Zürich, Suiza, al Dr. G. Guzmán del Instituto de Ecología, A.C., Xalapa, Veracruz, México y al Dr. G. Moreno de la Universidad de Alcalá, España, por sus valiosas críticas y sugerencias durante el desarrollo del trabajo doctoral. Se agradece a los curadores de los herbarios AH, FCME, GDAC, IBUG, LD, XAL, WTU y ZT por el préstamo de los materiales de Pluteus para su estudio.

\section{LITERATURA CITADA}

Banerjee, P. y W. Sundberg. 1993. Three new species and a new variety of Pluteus from the United States. Mycotaxon 47: 389-394.

Banerjee, P. y W. Sundberg. 1995. The genus Pluteus section Pluteus (Pluteaceae, Agaricales) in the midwestern United States. Mycotaxon 53: 189-246.

Breitenbach, J. y F. Kränzlin. 1995. Fungi of Switzerland. Agarics 2nd part. Vol. 4. Mykologia Lucerne. Lucerne. 368 pp.

Cifuentes, J., M. Villegas y L. Pérez-Ramírez. 1986. Hongos. In: Lot, A. y F. Chiang (comp.). Manual de herbarios. Consejo Nacional de la Flora de México. México, D.F. pp. 5564.

Consiglio, G. 1999. Contributo alla conoscenza del genere Pluteus. Boll. C. M. Carini 38: $1-12$.

Gartz, J. 1996. Magic mushroom around the world. A scientific journey across cultures and time. LIS publs. Los Angeles. 133 pp. 
Holmgren, P. K., N. H. Holmgren y L. C. Barnett (eds.). 1990. Index Herbariorum. Part I. The herbaria of the world. 8a. ed. New York Botanical Garden. Nueva York. 663 pp.

Kirk, P. M., P. F. Cannon, J. C. David y J. A. Stalpers. 2001. Ainsworth \& Bisby’s dictionary of the fungi. 9a. ed. CAB International. Wallingford. 655 pp.

Lange, J. E. 1936. Flora agaricina Danica Vol. I. Recato A/S Copenhage. Copenhage. 105 pp.

Largent, D. L. 1986. How to identify mushrooms to genus I: Macroscopic features. Mad River Press. Eureka. 166 pp.

Largent, D. L., D. Johnson y R. Watling. 1977. How to identify mushrooms to genus III: Microscopic features. Mad River Press. Eureka. 148 pp.

Murrill, W. A. 1917. Agaricaceae. North American Flora 10(2): 7-144.

Orton, P. D. 1986. Pluteaceae: Pluteus y Volvariella. In: Henderson, D. M., P. D. Orton y R. Watling (eds.). British fungus flora: Agarics and Boleti. Vol. 4. Royal Botanic Garden. Edinburgo. 99 pp.

Pegler, D. N. 1968. Studies on African Agaricales. I. Kew Bull. 21: 499-533.

Pegler, D. N. 1983. Agaric flora of the Lesser Antilles. Kew Bull. Add. Ser. IX. Her Majesty's Stat. Off. Londres. 668 pp.

Pradeep, C. K., K. B. Vrinda y T. K. Abraham. 2002. Pluteus section Pluteus (Pluteaceae, Agaricales) from Kerala State, India. Mycotaxon 83: 59-66.

Rodríguez, O. y L. Guzmán-Dávalos. 1997. New additions to the genus Pluteus (Pluteaceae, Agaricales) for Mexico. Micol. Neotrop. Apl. 10: 83-91.

Rodríguez, O. y L. Guzmán-Dávalos. 1998. Avances en el conocimiento del género Pluteus (Fungi, Agaricales) en Nueva Galicia, México. Bol. IBUG (Instituto de Botánica, Universidad de Guadalajara) 5(1-3): 87-93.

Rodríguez, O. y L. Guzmán-Dávalos. 2000. Algunas especies del género Pluteus (Pluteaceae, Agaricales) en Nueva Galicia, México. Bol. IBUG (Instituto de Botánica, Universidad de Guadalajara) 7(1-3): 61-77.

Rodríguez, O. y L. Guzmán-Dávalos. 2001. Clave dicotómica de las especies del género Pluteus Fr. (Pluteaceae) conocidas de la Nueva Galicia y algunas áreas aledañas, México. Acta Bot. Mex. 57: 23-36.

Rodríguez, O., L. Guzmán-Dávalos y O. Vargas. 1997. New reports of the genus Pluteus (Agaricales) from Mexico. Mycotaxon 61: 473-480.

Rodríguez, O., G. Moreno y M. Heykoop. 2004. El género Pluteus en México, diagnosis latinas y comentarios taxonómicos, corológicos y ecológicos. Bol. Soc. Micol. Madrid 28: 291-314.

Saupe, S. G. 1981. Occurrence of psilocybin/psilocin in Pluteus salicinus (Pluteaceae). Mycologia 73: 781-784.

Singer, R. 1958. Monographs of South American Basidiomycetes, especially those of the east slope of the Andes and Brazil. 1. The genus Pluteus in South America. Lloydia 21: 195-299.

Singer, R. 1973. Diagnoses fungorum novorum Agaricalium, III. Beih. Sydowia 7: 1-106.

Singer, R. 1989. New taxa and new combinations of Agaricales (Diagnoses fungorum novorum Agaricalium, IV). Fieldiana Botany 21: 1-33. 
Stijve, T. 1995. Worldwide occurrence of psychoactive mushroom-an update. Ceská Mykol. 48: $11-18$.

Stijve, T. y J. Bonnard. 1986. Psilocybine et urée dans le genre Pluteus. Mycol. Helv. 2: 123-130.

Ulloa, M. 1991. Diccionario ilustrado de micología. Universidad Nacional Autónoma de México, México, D.F. 310 pp.

Vellinga, E. C. 1988. Glossary. In: Bas, C., T. H. Kuyper, M. E. Noordeloos y E. C. Vellinga (eds.). Flora Agaricina Neerlandica Vol. 1. Balkema, Rotterdam. 182 pp.

Vellinga, E. C. 1990. Pluteaceae. In: Bas, C., T. H. Kuyper, M. E. Noordeloos y E. C. Vellinga (eds.). Flora Agaricina Neerlandica Vol. 2. Balkema, Rotterdam. 137 pp. 